\title{
Electric Field Induced Effects for Two-Electron Quantum Dot in Presence of Magnetic Field
}

\author{
A. PoszWA* \\ Faculty of Mathematics and Computer Science, University of Warmia and Mazury in Olsztyn, \\ Stoneczna 54, 10-710 Olsztyn, Poland
}

Received: 26.02.2020 \& Accepted: 07.05.2020

Doi: $10.12693 /$ APhysPolA.138.477

*e-mail: poszwa@matman. uwm.edu.pl

\begin{abstract}
We investigate the electric field induced Rashba coupling effect on spin- and magnetic properties of the system of two interacting electrons confined to a parabolic semiconductor quantum dot. The magnetic field $\boldsymbol{B}$ is applied perpendicularly to the plane of the dot. The total Hamiltonian of the system including Coulomb interaction between the electrons, the external $B$ field and the spin-orbit Rashba coupling is diagonalized using properly constructed basis. The electron correlation is taken into account exactly. The relative motion Schrödinger equation within the effective mass approximation has been solved by power series expansion method. The solutions are utilized for study the mechanism of mixing of two-electron spin states and to demonstrate how fundamental magnetic characteristics of the system reflecting B-field dependence of eigenenergies can be manipulated by means of electric field. Calculations are performed with material parameters of InSb quantum dot.
\end{abstract}

topics: Rashba spin-orbit coupling, electron correlation, magnetization

\section{Introduction}

Spin-orbit (SO) coupling in low-dimensional systems becomes recently the subject of extensive theoretical and experimental investigations. This is related to the key issue appearing in spintronics that is control of electron spin dynamics by means of external fields [1]. Due to SO interaction, the electron spin cannot be completely decoupled from its orbital motion. This fact has both positive and negative consequences. If the electron spin is completely decoupled from its orbital motion then the only way to influence it could be provided by an external magnetic field. As is well known, if an electron moves in an external electric field it experiences, in its rest frame, also a magnetic field. This leads to the effect known as the SO coupling. The effect has the origin in relativity. How large the effect is, depends on the intensity of the inner magnetic field experienced by the electron in its rest frame that is proportional to the external electric field. In solids, the electric fields responsible for the SO coupling are associated mainly with two asymmetries, the bulk inversion asymmetry and the structural inversion asymmetry. The latter arises from external confining potentials, created by materials interfaces or gate electrodes, giving rise to the so-called Rashba (Bychkov-Rashba) SO coupling [2-5]. In most of low-dimensional systems the Rashba coupling dominates other contributions to the SO interaction. The Rashba SO coupling extends greatly the abilities to control the spin dynamics since it allows for controlling the dynamics also by means of an electric field. However, the spin states influenced by the electric field may be devoid of phase coherence since SO interaction couples orthogonal orbital states with opposite spins. Even if the effect is small the spin quantum number is no longer a good quantum number and, until we are not interested either in particular positions of the particles nor in other degrees of freedom, the spin states could be described by means of the density matrix formalism $[6,7]$. The problem of phase coherence in systems with the SO coupling present has been recently studied and the relation of the information entropy to experimental characteristics of the spin was obtained $[8,9]$. A similar correspondence in the context of a scattering problem has been studied experimentally and the connection of the information entropy with the spin polarization has been demonstrated [10].

Many current investigations in semiconductor physics are devoted for the effect of SO coupling on various properties in different systems [11-17]. Physical processes related to disorder in spin-orbit coupling in two-dimensional electron gas with static and random sources of disorder have been investigated theoretically and comprehensively discussed in [18]. Stability of skyrmions in two-dimensional systems with the Rashba spin-orbit coupling has been obtained using a general analysis based on symmetry [19]. A spontaneous current production 
in the atomic thickness region near the interface between a superconductor and a ferromagnet has been recently predicted in superconducting systems with strong SO coupling [20]. The generalized approach to spin transport at interfaces with SO coupling has been proposed as a generalization of magnetoelectronic circuit theory [21-23]. The occupation number and the spin accumulation in parallel double dots with SO interaction have been studied theoretically by utilizing the Keldysh NEGF method [24]. Many other studies related to the spin have been performed on various properties and phenomena in quantum dots (QDs) including the magnetic field and Coulomb interactions [25-30]. The classical example is a sequence of singlet-triplet phase transitions [31-34] that appear as discontinuities in the magnetic properties of the dot [35]. The magnetization of non-circular QDs has been studied in the frame of the Hartree approximation [36]. Spin transport and magnetization dynamics in a quantum dot spin valve, i.e., two magnetic reservoirs coupled to a quantum dot have been studied taking into account effects of strong correlations [37, 38]. Quantum entanglement in Hooke's law atoms for different dimensionalities of the space has been studied in the frame of density matrix formalism [39]. The $B$ field dependence of the magnetization and the magnetic susceptibility for the two-electron InSb QD without SO interaction has been investigated [40]. One of the most important processes originated from SO interaction is the spin flips for the electron states in crystals without an inversion center [41]. The rates for the spin-flip transitions between the Zeeman sublevels in a quantum dot have been calculated by considering the mechanism of spin-phonon coupling via SO interaction [42]. The spin relaxation time $T_{1}$ (the time of a spin-flip process) and spin decoherence time $T_{2}$ (the lifetime of a coherent superposition of spin-up and spin-down states) in a GaAs quantum dot have been investigated for the Dresselhaus and Rashba SO couplings [43]. If the qubit is operated as a classical bit, its decay time is given by the spin relaxation time $T_{1}$. For quantum computation, however, the spin decoherence time $T_{2}$ must be sufficiently long. It has been shown in the paper [43] that the decoherence time $T_{2}$ of an electron spin in a GaAs QD is as large asthe relaxation time $T_{1}$ for the spin decay based on SO mechanisms.

In the present work we investigate the influence of the Rashba SO coupling and an external magnetic field on spin- and magnetic properties of the two-electron, parabolic QD. In particular, we investigate the Rashba SO coupling effect on mixing mechanism of two-electron spin states and on the magnetization and the magnetic susceptibility of the dot, using parameters appropriate for an InSb nanostructure. The Coulomb interaction between the electrons, the external $B$ field and the electron correlation are taken into account exactly in the sense that there are no approxima- tions made on the level of the Schrödinger equation related to relevant terms. All these contributions are taken into account simultaneously, for the first time. We note that due to a parabolic confinement, the two-electron wave functions can be found in the form of explicitly correlated functions (dependent explicitly on relative motion coordinates). Relative motion two-electron wave functions and energies are obtained in a frame of power series expansion method, that provides very accurate solutions $[34,44-46]$. The solutions are used to include the SO coupling effect, perturbatively. One should be also noted that the problem is treated as the two-dimensional one since the energy scale associated with confinement in the vertical direction is supposed to be very large compared to the energy scale, related to the lateral confinement. Under this assumption the dynamics may be considered as effectively two-dimensional one.

\section{Basic equations}

The system we study in this paper is the twodimensional, two-electron Rashba QD in the presence of a magnetic field. In the system the electrons are confined to a plane due to appropriate nanostructure. The electrostatic confining potential associated with a semiconductor structure forming the QD is supposed in the form of an asymmetric potential well producing nonzero gradient in the $z$-direction perpendicular to the plane of the dot. The zero-point energy has been chosen as confinement ground state energy of the quantum well. The confining asymmetric potential well is supposed as extremely narrow. Consequently, we confine ourselves to the one-band approximation. The potential is the source of the electric field that in turn is the source of the Rashba spin-orbit interaction. In general, the electric field may be the sum of an external field and the self-consistent electric field created by the nanostructure or gate electrodes. The situation is depicted in Fig. 1.

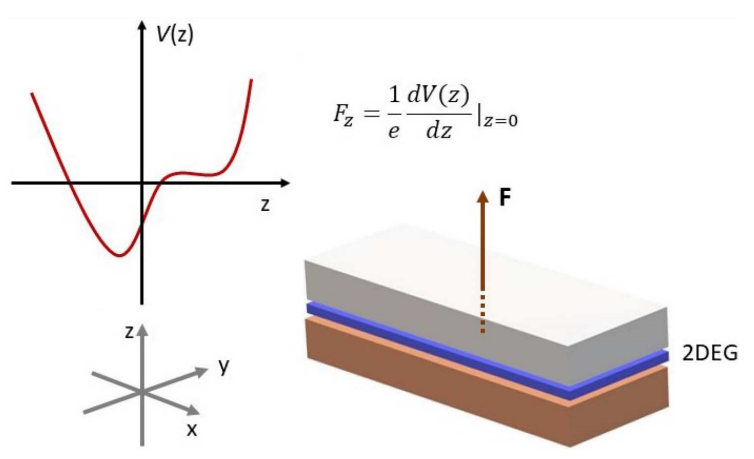

Fig. 1. Asymmetric semiconductor system hosting two-dimensional electron gas (2DEG). Confining potential associated with the structure is the source of the Rashba SO coupling. The QD can be produced by applying a lateral bias to a 2DEG leading to electron localization. 
In the presence of $\boldsymbol{B}$ field the Rashba SO coupling is described by the Hamiltonian

$$
\mathcal{H}_{\mathrm{SO}}=2 \frac{\boldsymbol{\eta}_{\mathrm{R}}}{\hbar} \cdot\left(\boldsymbol{s}_{1} \times \boldsymbol{\pi}_{1}+\boldsymbol{s}_{2} \times \boldsymbol{\pi}_{2}\right),
$$

where $\boldsymbol{\eta}_{\mathrm{R}}=\eta_{\mathrm{R}} \hat{\boldsymbol{z}}$ and $\eta_{\mathrm{R}}$ is the phenomenological Rashba parameter. Here, $m$ is the effective mass of the electron, $e$ is the absolute value of the electron charge. Supposing parabolic confinement with the strength $\omega_{0}$, the total Hamiltonian of two electrons confined to a $\mathrm{QD}$ reads

$$
\mathcal{H}_{\text {tot }}=\mathcal{H}_{0}+\mathcal{H}_{\mathrm{SO}} \text {, }
$$

where

$$
\begin{aligned}
\mathcal{H}_{0} & =\sum_{i=1}^{2}\left(\frac{1}{2 m} \boldsymbol{\pi}_{i}^{2}+\frac{1}{2} m \omega_{0}^{2} r_{i}^{2}\right)+\frac{e^{2}}{\epsilon_{r}\left|\boldsymbol{r}_{1}-\boldsymbol{r}_{2}\right|} \\
& +g^{*} \mu_{\mathrm{B}}\left(\boldsymbol{s}_{1}+\boldsymbol{s}_{2}\right) \cdot \boldsymbol{B},
\end{aligned}
$$

where $g^{*}$ denotes the effective Landé factor, $\mu_{\mathrm{B}}=e \hbar / 2 m_{e} c$ is the Bohr magneton, $\epsilon_{r}$ is the dielectric constant, $\boldsymbol{s}_{i}$ are dimensionless spin operators and

$$
\begin{aligned}
& \boldsymbol{\pi}_{i}=-\mathrm{i} \hbar \nabla_{i}+\frac{e}{c} \boldsymbol{A}\left(\boldsymbol{r}_{i}\right), \\
& \boldsymbol{A}(\boldsymbol{r})=\frac{1}{2} \boldsymbol{B} \times \boldsymbol{r} .
\end{aligned}
$$

One should be noted here that, for a free electron $g^{*}=2$, but for semiconductors we may have $g^{*}>0$, as well as $g^{*}<0$. Hereafter $\boldsymbol{B}=B \hat{\boldsymbol{z}}$. It is convenient for further analysis to introduce effective atomic units: $a_{B}=\frac{\hbar^{2}}{m e^{2}}$ as unit of length, $E_{h}=\frac{m e^{4}}{\hbar^{2}}$ as unit of energy, $B_{0}=\frac{m^{2} e^{3} c}{\hbar^{3}}$ as unit of magnetic field and the atomic unit of the Rashba coupling constant

$$
\eta_{0}=e^{2}=\alpha_{0} \hbar c \approx 1.43996 \mathrm{eV} \mathrm{nm},
$$

where $\alpha_{0}=\frac{e^{2}}{\hbar c} \approx 1 / 137$ is the fine structure constant. Note that since $\eta_{0}$ is independent of the electron mass and it determines the reference magnitude of the Rashba SO strength for arbitrary nanostructure. The Rashba parameter may vary by two orders of magnitude for various materials. In the case of InGaAs/InAlAs nanostructure $\quad \eta_{\mathrm{R}} \approx 0.67 \times 10^{-11} \mathrm{eV} \mathrm{m} \approx 0.005 \eta_{0}$ and for topological insulator $\mathrm{Bi}_{2} \mathrm{Se}_{3}$, $\eta_{\mathrm{R}} \approx 4 \times 10^{-10} \mathrm{eV} \mathrm{m} \sim 0.3 \eta_{0}[47]$.

The spin operators acting in the four-dimensional Hilbert space of the two-electron spin states are defined in the standard manner:

$$
s_{1}=\left(\frac{1}{2} \boldsymbol{\sigma}\right) \otimes \mathbb{I}, \quad \boldsymbol{s}_{2}=\mathbb{I} \otimes\left(\frac{1}{2} \boldsymbol{\sigma}\right),
$$

where $\mathbb{I}$ is the $2 \times 2$ unit matrix. The two-electron spin operators are given in Appendix A. By introducing centre-of-mass and relative motion coordinates

$$
\begin{aligned}
& \boldsymbol{R}=\{X, Y\}=\frac{1}{2}\left(\boldsymbol{r}_{1}+\boldsymbol{r}_{2}\right), \\
& \boldsymbol{r}=\{x, y\}=\boldsymbol{r}_{1}-\boldsymbol{r}_{2}
\end{aligned}
$$

and using effective atomic units we transform the Hamiltonian (3) to the form

$$
\mathcal{H}_{0}=\mathcal{H}_{\text {c.m. }}+\mathcal{H}_{\text {rel }}+\mathcal{H}_{\text {spin }},
$$

where

$$
\begin{aligned}
& \mathcal{H}_{\text {c.m. }}=-\frac{1}{4} \nabla_{\boldsymbol{R}}^{2}+\Omega^{2} \boldsymbol{R}^{2}-\frac{\mathrm{i}}{2} \gamma \frac{\partial}{\partial \phi} \\
& \mathcal{H}_{\text {rel }}=-\nabla_{\boldsymbol{r}}^{2}+\frac{1}{4} \Omega^{2} \boldsymbol{r}^{2}+\frac{1}{\epsilon_{r}|\boldsymbol{r}|}-\frac{\mathrm{i}}{2} \gamma \frac{\partial}{\partial \varphi}, \\
& \mathcal{H}_{\text {spin }}=\frac{1}{2} g \gamma \hat{S}_{z},
\end{aligned}
$$

where $\Omega=\sqrt{\Omega_{0}^{2}+\frac{1}{4} \gamma^{2}}, \Omega_{0}=\hbar \omega_{0} / E_{h}, \gamma=B / B_{0}$ and $g=g^{*}\left(m / m_{e}\right)$. The angles $\phi$ and $\varphi$ are polar angles corresponding to c.m. and relative motion coordinates, respectively. It is worth to point out that the scaling factor $\mathrm{m} / \mathrm{m}_{e}$ significantly lowers the spin contribution to the Zeeman shift, because of small effective mass comparing to the free electron mass. This contribution become important for systems with large absolute value of $g^{*}$, such as InSb nanostructure, for which $g^{*}=-51.56$.

Using coordinates (7) and the spin operators given in Appendix A, we can find matrix representation of the Hamiltonian (2). The SO part decouples into two terms,

$$
\mathcal{H}_{\mathrm{SO}}=\mathcal{H}_{\mathrm{SO}}^{\mathrm{c} . \mathrm{m} .}+\mathcal{H}_{\mathrm{SO}}^{\mathrm{rel}},
$$

where

$$
\begin{gathered}
\mathcal{H}_{\text {SO }}^{\text {c.m. }}=\alpha\left[\begin{array}{cccc}
0 & -\mathrm{e}^{-\mathrm{i} \phi} & -\mathrm{e}^{-\mathrm{i} \phi} & 0 \\
\mathrm{e}^{\mathrm{i} \phi} & 0 & 0 & -\mathrm{e}^{-\mathrm{i} \phi} \\
\mathrm{e}^{\mathrm{i} \phi} & 0 & 0 & -\mathrm{e}^{-\mathrm{i} \phi} \\
0 & \mathrm{e}^{\mathrm{i} \phi} & \mathrm{e}^{\mathrm{i} \phi} & 0
\end{array}\right] \frac{\partial}{\partial R} \\
-\alpha\left[\begin{array}{cccc}
0 & \mathrm{e}^{-\mathrm{i} \phi} & \mathrm{e}^{-\mathrm{i} \phi} & 0 \\
\mathrm{e}^{\mathrm{i} \phi} & 0 & 0 & \mathrm{e}^{-\mathrm{i} \phi} \\
\mathrm{e}^{\mathrm{i} \phi} & 0 & 0 & \mathrm{e}^{-\mathrm{i} \phi} \\
0 & \mathrm{e}^{\mathrm{i} \phi} & \mathrm{e}^{\mathrm{i} \phi} & 0
\end{array}\right] \hat{D}_{R},
\end{gathered}
$$

where $\alpha=\eta_{\mathrm{R}} / \eta_{0}$ and

$$
\hat{D}_{R}=\frac{1}{2} \gamma R-\frac{\mathrm{i}}{R} \frac{\partial}{\partial \phi} .
$$

The relative motion part of the SO Hamiltonian has a similar structure,

$$
\begin{gathered}
\mathcal{H}_{\text {SO }}^{\text {rel }}=\frac{\alpha}{2}\left[\begin{array}{cccc}
0 & \mathrm{e}^{-\mathrm{i} \varphi} & -\mathrm{e}^{-\mathrm{i} \varphi} & 0 \\
-\mathrm{e}^{\mathrm{i} \varphi} & 0 & 0 & -\mathrm{e}^{-\mathrm{i} \varphi} \\
\mathrm{e}^{\mathrm{i} \varphi} & 0 & 0 & \mathrm{e}^{-\mathrm{i} \varphi} \\
0 & \mathrm{e}^{\mathrm{i} \varphi} & -\mathrm{e}^{\mathrm{i} \varphi} & 0
\end{array}\right] \frac{\partial}{\partial r} \\
+\frac{\alpha}{2}\left[\begin{array}{cccc}
0 & \mathrm{e}^{-\mathrm{i} \varphi} & -\mathrm{e}^{-\mathrm{i} \varphi} & 0 \\
\mathrm{e}^{\mathrm{i} \varphi} & 0 & 0 & -\mathrm{e}^{-\mathrm{i} \varphi} \\
-\mathrm{e}^{\mathrm{i} \varphi} & 0 & 0 & \mathrm{e}^{-\mathrm{i} \varphi} \\
0 & -\mathrm{e}^{\mathrm{i} \varphi} & \mathrm{e}^{\mathrm{i} \varphi} & 0
\end{array}\right] \hat{D}_{r}, \quad(13)
\end{gathered}
$$

where

$$
\hat{D}_{r}=\frac{1}{2} \gamma r-\frac{\mathrm{i}}{r} \frac{\partial}{\partial \varphi} .
$$

To define good quantum numbers, we note that

$\left[\hat{S}^{2}, \mathcal{H}_{0}\right]=0, \quad\left[\hat{S}^{2}, \mathcal{H}_{\mathrm{SO}}^{\text {c.m. }}\right]=0$,

$$
\left[\hat{S}^{2}, \mathcal{H}_{\mathrm{SO}}^{\mathrm{rel}}\right] \neq 0,
$$


where $\hat{S}^{2}$ is the square of the total spin,

$$
\hat{S}^{2}=\left[\begin{array}{llll}
2 & 0 & 0 & 0 \\
0 & 1 & 1 & 0 \\
0 & 1 & 1 & 0 \\
0 & 0 & 0 & 2
\end{array}\right] \text {. }
$$

Therefore, in a case of the system described by the Hamiltonian $\mathcal{H}_{0}$, the total spin is conserved, whereas it is not conserved for the system with the SO interaction present. We can also see from commutation relations (15) that the total spin quantum number may be changed owing to the relative motion. The c.m. motion conserves the total spin. We can use eigenstates of $\mathcal{H}_{0}$ as the zeroorder approximation in the perturbation approach to the effect connected with SO coupling. We will confine ourselves to the first-order perturbation theory since the SO coupling constant for InSb structure is at most of the order $\approx 0.03[12,48]$. A comprehensive analysis of the second-order energy correction for one-electron Rashba QD may be found in [9].

In the case of any two-electron system a natural basis for spin states is the singlet-triplet basis. The basis has well-defined symmetry properties with respect to interchanging of the electrons. Taking this basis for the spin states, we can determine quantum numbers of spatial part of the total wave function to $\mathcal{H}_{0}$, according to the Pauli exclusion principle. By writing the total wave function of two electrons as $\Psi(1,2)=\Psi_{\text {c.m. }}(\boldsymbol{R}) \psi(\boldsymbol{r}) \chi(1,2)$ we can easily find that the operation of exchanging two electrons leads to $\Psi(2,1)=\Psi_{\text {c.m. }}(\boldsymbol{R}) \psi(-\boldsymbol{r}) \chi(2,1)$. Taking into account that the singlet (triplet) spin state is antisymmetric (symmetric) with respect to interchanging of the electrons, we have $\chi(2,1)=$ $(-1)^{S+1} \chi(1,2)$. Therefore, the total spin of two electrons confined to the $2 \mathrm{D}$ system with the circular symmetry is uniquely determined by the magnetic quantum number of the relative motion, according to the relation $(-1)^{S}=(-1)^{\left|m_{z}\right|}$. Hence, the singlet (triplet) spin states correspond to even (odd) $m_{z}$.

We look for eigenstates to the total Hamiltonian (2) as eigenstates of the projection of the total angular momentum

$$
\hat{J}_{z}=-\mathrm{i} \frac{\partial}{\partial \phi}-\mathrm{i} \frac{\partial}{\partial \varphi}+\hat{S}_{z}
$$

One can easily find that

$$
\left[\hat{J}_{z}, \mathcal{H}_{\text {tot }}\right]=0 \text {. }
$$

Therefore, the zero-order solution may be constructed in the form

$$
\begin{gathered}
|\Psi\rangle=C_{S}\left|\Phi_{S}\right\rangle+C_{+}\left|\Phi_{T_{+}}\right\rangle \\
+C_{0}\left|\Phi_{T_{0}}\right\rangle+C_{-}\left|\Phi_{T_{-}}\right\rangle,
\end{gathered}
$$

where we have introduced the following notations:

$$
\left|\Phi_{S}\right\rangle=\frac{\mathrm{e}^{\mathrm{i} M_{z} \phi}}{\sqrt{2 \pi}} \frac{\mathrm{e}^{\mathrm{i} m_{z} \varphi}}{\sqrt{2 \pi}} F_{N M_{z}}(R) G_{n m_{z}}(r)|00\rangle,
$$

where $m_{z}=0, \pm 2, \pm 4, \ldots$ and

$$
\begin{aligned}
& \left|\Phi_{T_{+}}\right\rangle=\frac{\mathrm{e}^{\mathrm{i} m_{z}^{\prime} \varphi}}{\sqrt{2 \pi}} \frac{\mathrm{e}^{\mathrm{i}\left(M_{z}+\Delta m_{z}-1\right) \phi}}{\sqrt{2 \pi}} \\
& \times G_{n^{\prime} m_{z}^{\prime}}(r) F_{N^{\prime}, M_{z}+\Delta m_{z}-1}(R)|11\rangle, \\
& \left|\Phi_{T_{0}}\right\rangle=\frac{\mathrm{e}^{\mathrm{i} m_{z}^{\prime} \varphi}}{\sqrt{2 \pi}} \frac{\mathrm{e}^{\mathrm{i}\left(M_{z}+\Delta m_{z}\right) \phi}}{\sqrt{2 \pi}} \\
& \quad \times G_{n^{\prime} m_{z}^{\prime}}(r) F_{N^{\prime}, M_{z}+\Delta m_{z}}(R)|10\rangle, \\
& \left|\Phi_{T_{-}}\right\rangle=\frac{\mathrm{e}^{\mathrm{i} m_{z}^{\prime} \varphi}}{\sqrt{2 \pi}} \frac{\mathrm{e}^{\mathrm{i}\left(M_{z}+\Delta m_{z}+1\right) \phi}}{\sqrt{2 \pi}} \\
& \quad \times G_{n^{\prime} m_{z}^{\prime}}(r) F_{N^{\prime}, M_{z}+\Delta m_{z}+1}(R)|1,-1\rangle,
\end{aligned}
$$

where $m_{z}^{\prime}= \pm 1, \pm 3, \ldots$ and $\Delta m_{z}=m_{z}-m_{z}^{\prime}$. Radial amplitudes and energies for c.m. and relative motion part, without the Coulomb interaction, are well known. They correspond to the $2 \mathrm{D}$ harmonic oscillator potential. For the case of interacting electrons solutions can be obtained using the power series expansion method. Radial amplitudes corresponding to both non-interacting and interacting case are given in Appendix B. We note that the states (20) and (21) correspond to the same eigenvalue of $\hat{J}_{z}$, equal to $M_{z}+m_{z}$. In the triplet sector, the radial amplitudes may have, in general, different quantum numbers $\left(n^{\prime}, N^{\prime}\right)$. However, we have limited ourselves to the same pairs of the quantum numbers since overlap integrals between radial functions strongly decrease with increasing difference between radial quantum numbers.

Using for spin states the basis $\mathrm{B} 2=\left\{S, T_{+}, T_{0}, T_{-}\right\}$ (see Appendix A) we can directly obtain matrix representation for $H_{0}$. We can write it as the sum of three terms,

$$
\left[\mathcal{H}_{0}\right]=\left[\mathcal{H}_{\text {c.m. }}\right]+\left[\mathcal{H}_{\text {rel }}\right]+\left[\mathcal{H}_{\text {spin }}\right],
$$

where all matrices have diagonal forms,

$$
\begin{aligned}
& {\left[\mathcal{H}_{\mathrm{c} . \mathrm{m} .}\right]=\operatorname{diag}\left[E_{N M_{z}}, E_{N^{\prime}, M_{z}+\Delta m_{z}-1},\right.} \\
& \left.E_{N^{\prime}, M_{z}+\Delta m_{z}}, E_{N^{\prime}, M_{z}+\Delta m_{z}+1}\right], \\
& {\left[\mathcal{H}_{\mathrm{rel}}\right]=\operatorname{diag}\left[E_{n m_{z}}, E_{n^{\prime} m_{z}^{\prime}}, E_{n^{\prime} m_{z}^{\prime}}, E_{n^{\prime} m_{z}^{\prime}}\right],}
\end{aligned}
$$
where $E_{N M_{z}}$ and $E_{n m_{z}}$ denote the c.m. and relative motion energies, respectively, and

$$
\left[\mathcal{H}_{\text {spin }}\right]=\frac{1}{2} \gamma g \operatorname{diag}[0,1,0,-1] .
$$

After some algebraic calculations, the SO Hamiltonian matrix is found in the form

$$
\left.\left[\mathcal{H}_{\mathrm{SO}}\right]=\left[\begin{array}{cccc}
0 & \mathbf{\square}_{\text {rel }} & 0 & \boldsymbol{\Delta}_{\text {rel }} \\
\mathbf{\square}_{\text {rel }} & 0 & \square_{\text {c.m. }} & 0 \\
0 & \square_{\text {c.m. }} & 0 & \nabla_{\text {c.m }} \\
\mathbf{\Delta}_{\text {rel }} & 0 & \nabla_{\text {c.m }} & 0
\end{array}\right]\right\} \mathrm{S} \leftrightarrow \mathrm{T}
$$

On the right we indicated blocks responsible for coupling between relevant spin states. The entries of the matrix are given in Appendix C. Mixing of different spin states due to $\mathrm{SO}$ interaction is illustrated graphically in Fig. 2. One can see again that 


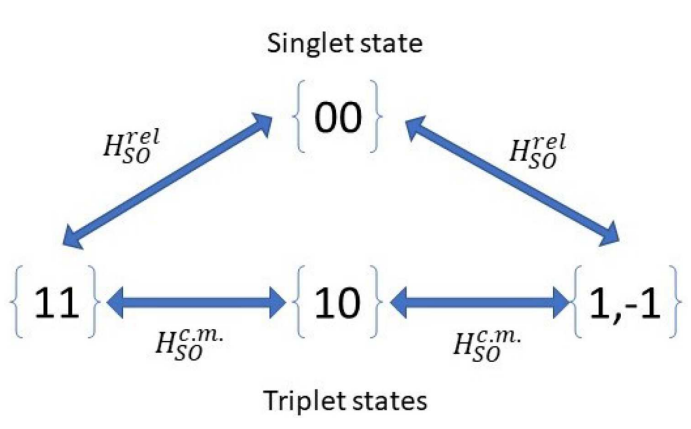

Fig. 2. Mixing of two-electron spin states due to SO coupling.

the c.m. motion does not change the total spin. The c.m. motion mixes only appropriate triplet spin states.

The Schrödinger equation for the two-electron Rashba QD,

$$
\mathcal{H}_{\text {tot }}|\Psi\rangle=E|\Psi\rangle
$$

has the matrix representation

$$
\left[\mathcal{H}_{\text {tot }}\right]\{C\}=E\{C\} \text {, }
$$

where $\left[\mathcal{H}_{\text {tot }}\right]=\left[\mathcal{H}_{0}\right]+\left[\mathcal{H}_{\mathrm{SO}}\right]$ and $\{C\}=$ $\left[C_{S}, C_{+}, C_{0}, C_{-}\right]^{\mathrm{T}}$. Solving the last algebraic equation we can determine four levels having the same projection of the total angular momentum.

\section{The magnetization operator}

In order to study magnetic properties of the system composed from certain number of charged particles $N_{p}$, it is convenient to introduce the magnetization operator. The magnetic moment of the system (the magnetization) may be obtained by averaging the magnetization operator. According to [49] we defined the magnetization operator:

$$
\begin{gathered}
\boldsymbol{M}=-\frac{e}{2 m c} \sum_{i=1}^{N_{p}} \boldsymbol{r}_{i} \times\left(\boldsymbol{p}_{i}+\frac{e}{c} \boldsymbol{A}\left(\boldsymbol{r}_{i}\right)\right) \\
-g \frac{e \hbar}{2 m c} \sum_{i=1}^{N_{p}} \mathbf{s}_{i},
\end{gathered}
$$

where, in order to include both the orbital and the spin magnetization, we have supplemented the operator defined in [49] by the spin term. For a uniform magnetic field one obtains

$$
\begin{array}{r}
\boldsymbol{M}=-\frac{e \hbar}{2 m c} \sum_{i=1}^{N_{p}}\left(\boldsymbol{l}_{i}+g \boldsymbol{s}_{i}\right) \\
-\frac{e^{2}}{4 m c^{2}} \boldsymbol{B} \sum_{i=1}^{N_{p}}\left(x_{i}^{2}+y_{i}^{2}\right),
\end{array}
$$

where operators $\boldsymbol{l}_{i}$ and $\boldsymbol{s}_{i}$ are dimensionless. The magnetic moment of the system gives the $\boldsymbol{B}$ dependent contribution

$$
\mathrm{d} \mathcal{H}^{\prime}=-\boldsymbol{M} \cdot \mathrm{d} \boldsymbol{B} .
$$

An alternative expression for the Hamiltonian $\mathcal{H}_{0}$ in terms of $\boldsymbol{M}$, reads

$$
\mathcal{H}_{0}=\sum_{i=1}^{N_{p}} \frac{p_{i}^{2}}{2 m}-\int \boldsymbol{M} \cdot \mathrm{d} \boldsymbol{B}+V_{\text {conf }}+V_{\mathrm{C}},
$$

where the potentials $V_{\text {conf }}=\frac{m \omega^{2}}{2}\left(r_{1}^{2}+r_{2}^{2}\right)$ and $V_{\mathrm{C}}=\frac{1}{\epsilon_{r}} \frac{e^{2}}{\boldsymbol{r}_{1}-\boldsymbol{r}_{2}}$ are $B$ independent. The magnetization operator reads as

$$
\boldsymbol{\mu}=-\frac{1}{2} \sum_{i=1}^{N_{p}}\left(\boldsymbol{l}_{i}+g \boldsymbol{s}_{i}\right)-\frac{1}{4} \frac{\boldsymbol{B}}{B_{0}} \sum_{i=1}^{N_{p}}\left(x_{i}^{2}+y_{i}^{2}\right)
$$

in units of $[e \hbar / m c]$ (two effective Bohr magnetons). We can also express the magnetization in units of $[\mathrm{meV} / \mathrm{T}]$ according to the relation

$$
\left[\frac{e \hbar}{m c}\right]=\frac{E_{h}}{B_{0}}=\frac{0.27211}{2.35} \frac{m_{e}}{m}\left[\frac{\mathrm{meV}}{\mathrm{T}}\right] .
$$

In the case of a planar system with circular symmetry, placed in a magnetic field oriented along the $z$-axis, the only non-zero component of the magnetization vector $\boldsymbol{\mu}$ is its $z$-component, $\mu_{z}$. Using the c.m. and relative motion coordinates $\left(N_{p}=2\right)$ we obtain

$$
\begin{gathered}
\mu_{z}=-\frac{1}{2}\left\langle\Psi\left|\hat{J}_{z}\right| \Psi\right\rangle+\frac{1-g}{2}\left\langle\Psi\left|\hat{S}_{z}\right| \Psi\right\rangle \\
-\frac{1}{2} \gamma\left(\left\langle\Psi\left|R^{2}\right| \Psi\right\rangle+\frac{1}{4}\left\langle\Psi\left|r^{2}\right| \Psi\right\rangle\right) .
\end{gathered}
$$

For the state vector $|\Psi\rangle$ given by (19) one obtains

$$
\begin{aligned}
\mu_{z} & =-\frac{1}{2}\left(M_{z}+m_{z}\right)+\frac{1-g}{2}\left(C_{+}^{2}-C_{-}^{2}\right) \\
& -\frac{1}{2} \gamma\left(\left\langle\Psi\left|R^{2}\right| \Psi\right\rangle+\frac{1}{4}\left\langle\Psi\left|r^{2}\right| \Psi\right\rangle\right) .
\end{aligned}
$$

Using the virial theorem one can easily find that in any state of the c.m. motion

$$
\left\langle N M_{z}\left|R^{2}\right| N M_{z}\right\rangle=\frac{1}{2 \Omega}\left(2 N+M_{z}+1\right) .
$$

In a similar way may be calculated the second matrix element in the last term of $\mu_{z}$,

$$
\left\langle n m_{z}\left|r^{2}\right| n m_{z}\right\rangle=\frac{2}{\Omega}\left(2 n+m_{z}+1\right) .
$$

In the case including the Coulomb interaction, the average value of $r^{2}$ is calculated numerically, using approximate wave functions (see Appendix B).

\section{Results and discussion}

We studied the effect of electric-field induced SO coupling in the presence of the external $B$ field for two interacting electrons confined to the semiconductor QD, within the effective mass approximation. We shown that the SO coupling significantly affects spin-dependent properties of the dot. We investigated the influence of the coupling on the magnetization and magnetic susceptibility of the dot as functions of external magnetic field and the Rashba SO coupling strength. We showed how mixing of two-electron spin states is correlated with the c.m. motion and the relative motion. To reference our analysis to a realistic world, the parameters of the model were fixed on values corresponding to the InSb nanostructure. 
TABLE I

Relative motion energies $E_{\text {rel }}$ (in meV) of two-electron InSb QD $\left(m=0.0139 m_{e}, \epsilon_{r}=14\right)$ compared with energies of noninteracting electrons $E_{\mathrm{rel}}^{(0)}$, for different confining energies. The contribution from the Coulomb interaction is given by $\delta=\left(E_{\mathrm{rel}}-E_{\mathrm{rel}}^{(0)}\right) / E_{\mathrm{rel}}^{(0)}$ (in \%). The magnetic field $\gamma=0$.

\begin{tabular}{c|c|c|c|c|c|c|c|c|c|c}
\hline \hline \multirow{2}{*}{$m_{z}$} & \multirow{2}{*}{$n$} & \multicolumn{3}{|c|}{$\hbar \omega_{0}=4 \mathrm{meV}$} & \multicolumn{3}{c|}{$\hbar \omega_{0}=8 \mathrm{meV}$} & \multicolumn{3}{c}{$\hbar \omega_{0}=20 \mathrm{meV}$} \\
\cline { 3 - 10 } & & $E_{\text {rel }}$ & $E_{\text {rel }}^{(0)}$ & $\delta$ & $E_{\text {rel }}$ & $E_{\text {rel }}^{(0)}$ & $\delta$ & $E_{\text {rel }}$ & $E_{\text {rel }}^{(0)}$ & $\delta$ \\
\hline 0 & 0 & 6.94761018 & 4.00000000 & 73.7 & 12.3554673 & 8.00000000 & 54.4 & 27.1836969 & 20.0000000 & 35.9 \\
& 1 & 14.4316019 & 12.0000000 & 20.3 & 27.5145868 & 24.0000000 & 14.6 & 65.6633485 & 60.0000000 & 9.4 \\
& 2 & 22.1377351 & 20.0000000 & 10.7 & 43.0647893 & 40.0000000 & 7.7 & 104.901463 & 100.000000 & 4.9 \\
\hline 1 & 0 & 9.68139008 & 8.00000000 & 21.0 & 18.4015478 & 16.0000000 & 15.0 & 43.8315190 & 40.0000000 & 9.6 \\
& 1 & 17.4923934 & 16.0000000 & 9.3 & 34.1232558 & 32.0000000 & 6.6 & 83.3751502 & 80.0000000 & 4.2 \\
& 2 & 25.3678468 & 24.0000000 & 5.7 & 49.9425297 & 48.0000000 & 4.1 & 123.082778 & 120.000000 & 2.6 \\
\hline 2 & 0 & 13.2848780 & 12.0000000 & 10.7 & 25.8255773 & 24.0000000 & 7.6 & 62.8985870 & 60.0000000 & 4.8 \\
& 1 & 21.1836273 & 20.0000000 & 5.9 & 41.6793728 & 40.0000000 & 4.2 & 102.663060 & 100.000000 & 2.7 \\
& 2 & 29.1093292 & 28.0000000 & 4.0 & 57.5727170 & 56.0000000 & 2.8 & 142.492161 & 140.000000 & 1.8
\end{tabular}
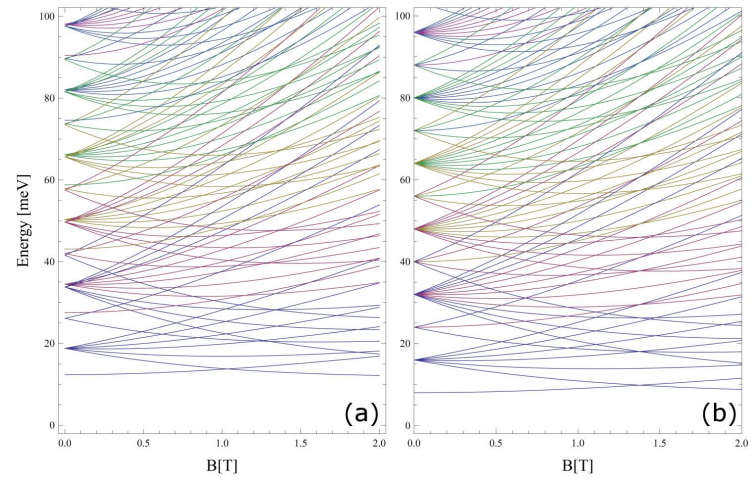

Fig. 3. Dependence on the $B$ field of lowest relative motion levels including spin $(n=0, \ldots 10$, $\left.m_{z}=0, \pm 1, \pm 2, \pm 3, \pm 4, S_{z}=0, \pm 1\right)$ of two-electron QD: (a) interacting electrons, (b) noninteracting electrons. Confining energy $\hbar \omega_{0}=8 \mathrm{meV}$. InSb material parameters are used: $m=0.0139 m_{e}$ $g^{*}=-51.56, \epsilon_{r}=14$.

In Fig. 3a and b there are compared relative motion energy spectra for systems with the Coulomb interaction present and with the Coulomb interaction neglected, respectively. The SO coupling is not apparent at this scale. In general, the Coulombic repulsion upshifts the energy levels and moves crossings of levels towards lower magnetic fields.

In Table I there are given relative motion energies of the two-electron QD for increasing confining energies $\hbar \omega_{0}$. Energies are computed with the accuracy of 9 decimal figures. We also display the relative Coulomb contribution (including electron correlation) given by $\delta$. We can see that the role of the Coulomb interaction is very important. One can see that for low-lying states, the relative Coulomb contribution is greater that for higher states. In particular, for ground state $\delta \approx 74 \%$. This means that the Coulomb interaction strongly affects low-temperature properties of the dot, such

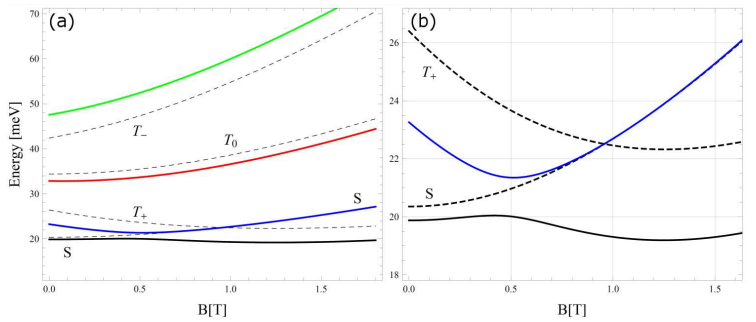

Fig. 4. Levels $\left(N=0, M_{z}=0, n=0, m_{z}=0\right.$, $\left.n^{\prime}=0, m_{z}^{\prime}=-1\right)$ of the two-electron InSb QD: (a) with SO coupling (solid lines) and without SO coupling (dashed lines), (b) levels crossing (dashed lines) and avoiding crossing (solid lines). Confining energy $\hbar \omega_{0}=8 \mathrm{meV}$, the Rashba coupling constant $\alpha=0.05$.

as the magnetization, which are dominated by lowlying states. We can see that the relative contribution of the electron-electron interaction decreases with increasing both the angular momentum quantum number $m_{z}$ and the radial quantum number $n$. This follows directly from the behavior of radial wave functions at small distances.

In Fig. 4a there is given dependence on the $B$ field of four levels $S, T_{+}, T_{0}, T_{-}$, in the basis of which the total Hamiltonian is diagonalized. The levels are plotted as dashed lines. On the other hand, levels, plotted as solid lines, correspond to eigenstates of the total Hamiltonian. Two higher levels are simply shifted relatively to $T_{-}$and $T_{0}$, by approximately constant amounts of opposite signs. In part (b) there are given two lowest levels that are mainly a mixture of the singlet $S$ and the triplet $T_{+}$ states. The states are strongly mixed in the vicinity of the transition point. We note that the singlettriplet anticrossings in two-electron quantum dots are used as one of the ways for experimental observations of SO interaction, e.g. see the experimental work on two-electron self-assembled InAs quantum dots [50]. 


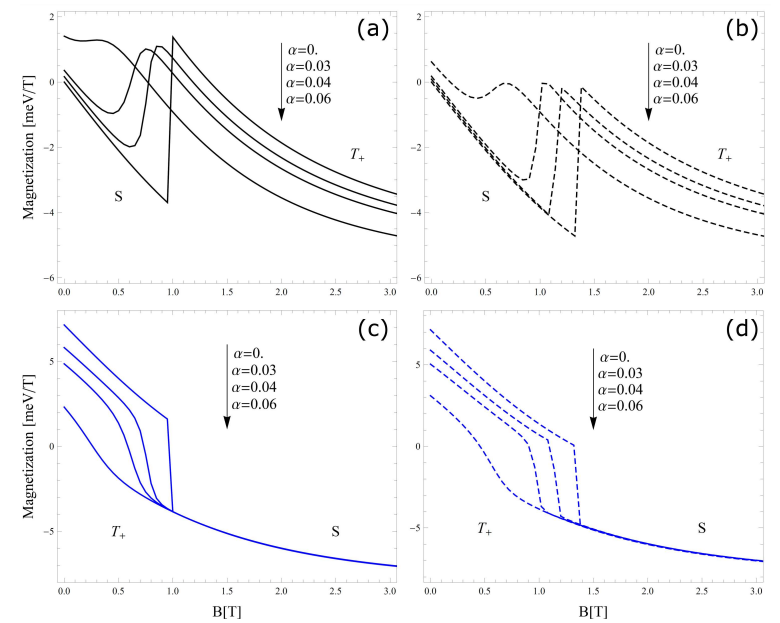

Fig. 5. Dependence on $B$ field of magnetization of InSb QD: (a) ground state with e-e interaction, (b) ground state without e-e interaction, (c) first excited state with e-e interaction, (d) first excited state without e-e interaction. Confining energy $\hbar \omega_{0}=8 \mathrm{meV}$. Symbols $S, T_{+}$refer to regions with dominating states $S, T_{+}$, respectively.

In Fig. 5 there is given the dependence of the magnetic moment of the dot (the $z$-component of the magnetization $\boldsymbol{\mu}(B))$ on the $B$ field. In the absence of the Rashba coupling, crossings of $S$ and $T_{+}$levels appear as discontinuities in the magnetization. Due to the SO coupling, the jump in the magnetization becomes gradual. One should be noted that the role of the Coulomb interaction is significant. In general, the interaction upshifts the ground state magnetization to positive values. As we can see in part (a), the magnetization may have both positive and negative values around the transition point while if the Coulomb interaction is absent (part (b)) the ground state magnetization is negative. The presence of both signs means the possibility of changing the magnetic moment of the dot, from an initial sign to the opposite one, by means of the magnetic field. In the presence of the electric field the process is continuous. This property may have importance for the practical implementations of QDs in fabrication of devices based on the magnetization, such as magnetoresistance sensors or other devices using the magnetization reversal (switching) [51]. We note that in the $\alpha \approx 0$ limit our result calculated for confining energy $\hbar \omega_{0}=8 \mathrm{meV}$ fundamentally agrees with the result by De Groote et al. [40], obtained for the two-electron InSb QD in the absence of SO coupling, with a little different material parameters and for the confining energy $\hbar \omega_{0}=7.5 \mathrm{meV}$. In parts (c) and (d) there is given the magnetization in the first excited state of the dot. The magnetization reveals the saturation with increasing $B$ field. Using (36)-(38) we can find that $\lim _{\gamma \rightarrow \infty} \mu_{z}=-1$. Taking into account (34) one obtains the saturation value, $\approx-8.6 \mathrm{meV} / \mathrm{T}$.

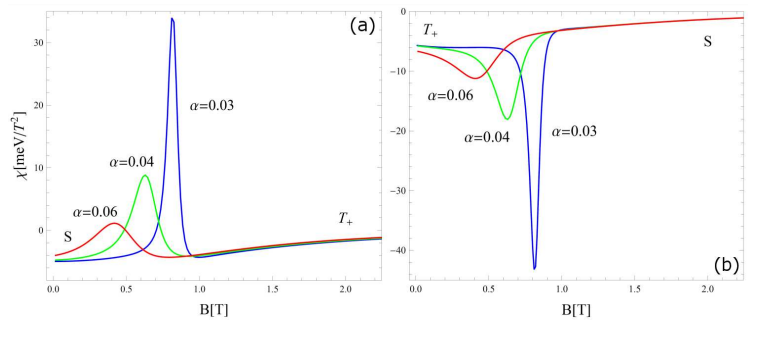

Fig. 6. Magnetic susceptibilities of InSb QD for different Rashba coupling constants: (a) ground state, (b) first excited state. Confining energy $\hbar \omega_{0}=8 \mathrm{meV}$. Symbols $S, T_{+}$refer to regions with dominating states $S, T_{+}$, respectively.
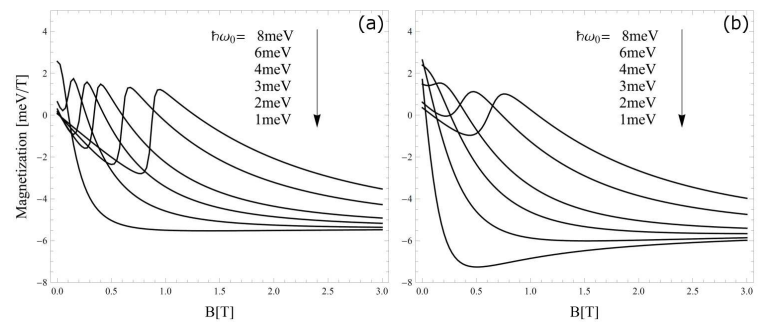

Fig. 7. Ground-state magnetization of InSb QD as function of confining energy for the Rashba coupling strength: (a) $\alpha=0.02$, (b) $\alpha=0.04$.

The behavior of the magnetization in function of the $B$ field has relevant consequences for the magnetic susceptibility of the dot. At temperature $T=0$, the magnetic susceptibility can be defined as $\chi=\mathrm{d} \mu_{z} / \mathrm{d} \gamma$. We can see in Fig. 6 that there are created pertinent domains with well-defined signs of the magnetic susceptibility. In the case of first excited state, the magnetic susceptibility $\chi<0$ for every $B$ field and the dot, in this state, is a diamagnetic.

In Fig. 7 there is given dependence of the magnetization curves for increasing confinement. One can see that for low confinement, crossings of corresponding levels are damped. In a consequence, the magnetization curves are regular. For higher confinement, the magnetization discloses decreasing jump with increasing Rashba coupling strength.

\section{Summary}

We have presented a detailed theoretical study of the SO coupling effect induced by self-consistent electric field created by the nanostructure for two interacting electrons confined to a parabolic QD, using semiconductor InSb QD. We note that the twoelectron problem including simultaneously e-e interaction, electron correlation, external $B$ field and SO interaction is studied for the first time. As well known the two-electron problem in a parabolic potential in a magnetic field has a unique property of separability the relative motion from the c.m. motion. The presence of an external electric field perpendicular to the plane of the system does not affect 
this property. This allows for taking into account the electron correlation exactly also for the system in the SO interaction present. The solution to the relative motion Schrödinger equation including the Coulomb interaction and external $B$ field has been obtained by power series expansion method in combination with the iteration of asymptotic boundary conditions imposed on the radial function at finite distance. Using this solution we have shown that both electron-electron interaction and electron correlation have important consequences for spin-dependent properties of the dot in the SO coupling present. One should be also pointed out that since we can control the spin-orbit coupling constant in planar semiconductor systems by means of external or built-in electric fields, we can change, by variations of the fields, the magnetic properties of the system. The precise controlling of the magnetization of the system is of particular interest from the point of view of spintronic devices realization involving magnetic contacts and manipulated by electric fields. The exploration of the Rashba physics is now at the heart of the growing research field of spin-orbitronics, a branch of spintronics focusing on the manipulation of non-equilibrium materials properties using SO coupling. Obtained results may therefore find some applications in experimental investigations of the Rashba SO coupling.

\section{APPENDIX A: Two-electron spin operators}

The spin operators corresponding to the total spin of two electrons may be obtained as the sum of single particle spin operators,

$$
\hat{\boldsymbol{S}}=\boldsymbol{s}_{1}+\boldsymbol{s}_{2} \text {. }
$$

Using standard representation, in which singleelectron spin operators $s^{2}$ and $s_{z}$ are diagonal, referred to as B1 representation, we obtain immediately the two-electron spin matrices, by calculating

$$
\hat{\boldsymbol{S}}=\left(\frac{1}{2} \boldsymbol{\sigma}\right) \otimes \mathbb{I}+\mathbb{I} \otimes\left(\frac{1}{2} \boldsymbol{\sigma}\right) .
$$

The two-electron spin operators in the B1 representation have forms

$$
\begin{aligned}
& \hat{S}_{x}=\frac{1}{2}\left[\begin{array}{llll}
0 & 1 & 1 & 0 \\
1 & 0 & 0 & 1 \\
1 & 0 & 0 & 1 \\
0 & 1 & 1 & 0
\end{array}\right], \hat{S}_{y}=\frac{1}{2}\left[\begin{array}{cccc}
0 & -\mathrm{i} & -\mathrm{i} & 0 \\
\mathrm{i} & 0 & 0 & -\mathrm{i} \\
\mathrm{i} & 0 & 0 & -\mathrm{i} \\
0 & \mathrm{i} & \mathrm{i} & 0
\end{array}\right], \\
& \hat{S}_{z}=\left[\begin{array}{llll}
1 & 0 & 0 & 0 \\
0 & 0 & 0 & 0 \\
0 & 0 & 0 & 0 \\
0 & 0 & 0 & -1
\end{array}\right] .
\end{aligned}
$$

The square of the total spin in the B1 representation reads

$$
\hat{S}^{2}=\hat{S}_{x}^{2}+\hat{S}_{y}^{2}+\hat{S}_{z}^{2}=\left[\begin{array}{cccc}
2 & 0 & 0 & 0 \\
0 & 1 & 1 & 0 \\
0 & 1 & 1 & 0 \\
0 & 0 & 0 & 2
\end{array}\right]
$$

We define new basis, referred to as $\mathrm{B} 2=\left\{S, T_{+}, T_{0}, T_{-}\right\}$, where

$|S\rangle \equiv|00\rangle=$

$\frac{1}{\sqrt{2}}\left\{\left[\begin{array}{l}1 \\ 0\end{array}\right] \otimes\left[\begin{array}{l}0 \\ 1\end{array}\right]-\left[\begin{array}{l}0 \\ 1\end{array}\right] \otimes\left[\begin{array}{l}1 \\ 0\end{array}\right]\right\}=\frac{1}{\sqrt{2}}\left[\begin{array}{c}0 \\ 1 \\ -1 \\ 0\end{array}\right]$,

$\left|T_{0}\right\rangle \equiv|10\rangle=$

$\frac{1}{\sqrt{2}}\left\{\left[\begin{array}{l}1 \\ 0\end{array}\right] \otimes\left[\begin{array}{l}0 \\ 1\end{array}\right]+\left[\begin{array}{l}0 \\ 1\end{array}\right] \otimes\left[\begin{array}{l}1 \\ 0\end{array}\right]\right\}=\frac{1}{\sqrt{2}}\left[\begin{array}{l}0 \\ 1 \\ 1 \\ 0\end{array}\right]$,

$$
\begin{aligned}
& \left|T_{+}\right\rangle \equiv|11\rangle=\left[\begin{array}{l}
1 \\
0
\end{array}\right] \otimes\left[\begin{array}{l}
1 \\
0
\end{array}\right]=\left[\begin{array}{l}
1 \\
0 \\
0 \\
0
\end{array}\right], \\
& \left|T_{-}\right\rangle \equiv|1,-1\rangle=\left[\begin{array}{l}
0 \\
1
\end{array}\right] \otimes\left[\begin{array}{l}
0 \\
1
\end{array}\right]=\left[\begin{array}{l}
0 \\
0 \\
0 \\
1
\end{array}\right] .
\end{aligned}
$$

The basis B1 can be obtained from the basis B2 by the unitary transformation, given by

$$
U=\left[\begin{array}{cccc}
0 & 1 & 0 & 0 \\
\frac{1}{\sqrt{2}} & 0 & \frac{1}{\sqrt{2}} & 0 \\
-\frac{1}{\sqrt{2}} & 0 & \frac{1}{\sqrt{2}} & 0 \\
0 & 0 & 0 & 1
\end{array}\right]
$$

The spin operators in the $\mathrm{B} 2$ representation read

$$
\begin{aligned}
& \hat{S}^{2}=U^{\dagger} \hat{S}^{2} U=\left[\begin{array}{llll}
0 & 0 & 0 & 0 \\
0 & 2 & 0 & 0 \\
0 & 0 & 2 & 0 \\
0 & 0 & 0 & 2
\end{array}\right], \\
& \hat{S}_{z}=U^{\dagger} \hat{S}_{z} U=\left[\begin{array}{llll}
0 & 0 & 0 & 0 \\
0 & 1 & 0 & 0 \\
0 & 0 & 0 & 0 \\
0 & 0 & 0 & -1
\end{array}\right], \\
& \hat{S}_{x}=U^{\dagger} \hat{S}_{x} U=\left[\begin{array}{cccc}
0 & 0 & 0 & 0 \\
0 & 0 & \frac{1}{\sqrt{2}} & 0 \\
0 & \frac{1}{\sqrt{2}} & 0 & \frac{1}{\sqrt{2}} \\
0 & 0 & \frac{1}{\sqrt{2}} & 0
\end{array}\right], \\
& \hat{S}_{y}=U^{\dagger} \hat{S}_{y} U=\left[\begin{array}{cccc}
0 & 0 & 0 & 0 \\
0 & 0 & \frac{-\mathrm{i}}{\sqrt{2}} & 0 \\
0 & \frac{\mathrm{i}}{\sqrt{2}} & 0 & \frac{-\mathrm{i}}{\sqrt{2}} \\
0 & 0 & \frac{\mathrm{i}}{\sqrt{2}} & 0
\end{array}\right],
\end{aligned}
$$

where we have used the same symbols for both representations. 


\section{APPENDIX B: Radial amplitudes}

\section{B.1. Centre-of-mass motion}

Solutions to the Schrödinger equation for the c.m. motion

$$
\mathcal{H}_{\text {c.m }} \Psi_{\text {c.m }}=E_{\text {c.m }} \Psi_{\text {c.m }},
$$

may be found analytically. The normalized solutions have forms

$$
\Psi_{\text {c.m }}(R, \phi)=\frac{\mathrm{e}^{\mathrm{i} M_{z} \phi}}{\sqrt{2 \pi}} F_{N M_{z}}(R),
$$

where the radial amplitude reads

$$
\begin{gathered}
F_{N M_{z}}(R)=\mathcal{N}_{N M_{z}} R^{M_{z}} \mathrm{e}^{-\Omega R^{2}} \\
\times F\left(-N, M_{z}+1,2 \Omega R^{2}\right),
\end{gathered}
$$

where $F$ is confluent hypergeometric function and

$$
\mathcal{N}_{N M_{z}}=\frac{2}{M_{z} !} \sqrt{\frac{2^{M_{z} \Omega^{M_{z}+1} \Gamma\left(N+M_{z}+1\right)}}{N !}} .
$$

Energies are given by

$$
E_{\mathrm{c} . \mathrm{m}}=\left(2 N+M_{z}+1\right) \Omega+\frac{1}{2} \gamma M_{z} .
$$

In the main text, the c.m. energies are denoted as $E_{N M_{z}}$.

\section{B.2. Relative motion}

\section{B.2.1. The case of noninteracting electrons}

The Schrödinger equation for noninteracting electrons reads

$$
\mathcal{H}_{\mathrm{rel}}^{(0)} \Psi_{\mathrm{rel}}^{(0)}=E_{\mathrm{rel}}^{(0)} \Psi_{\mathrm{rel}}^{(0)}
$$

where

$$
\mathcal{H}_{\text {rel }}^{(0)}=-\Delta+\frac{1}{4} \Omega^{2} r^{2}+\frac{1}{2} \gamma m_{z} .
$$

The normalized solutions have the form

$$
\Psi_{\text {rel }}^{(0)}(r, \varphi)=\mathcal{N}_{n m_{z}} \frac{\mathrm{e}^{\mathrm{i} m_{z} \varphi}}{\sqrt{2 \pi}} G_{n m_{z}}^{(0)}(r),
$$

where radial amplitudes read

$$
\begin{aligned}
& G_{n m_{z}}^{(0)}(r)=\mathcal{N}_{n m_{z}} r^{m_{z}} \mathrm{e}^{-\Omega r^{2} / 4} \\
& \quad \times F\left(-n, m_{z}+1, \frac{1}{2} \Omega r^{2}\right),
\end{aligned}
$$

where

$$
\mathcal{N}_{n m_{z}}=\frac{\sqrt{2^{-m_{z} \Omega^{m_{z}+1} \Gamma\left(n+m_{z}+1\right)}}}{m_{z} ! \sqrt{n !}} .
$$

Energies are given by

$$
E_{\mathrm{rel}}^{(0)}=\left(2 n+m_{z}+1\right) \Omega+\frac{1}{2} \gamma m_{z} .
$$

In the main text, both the analytic and approximate relative motion energies are denoted as $E_{n m_{z}}$.

\section{B.2.2. The case of interacting electrons}

The Schrödinger equation for the relative motion reads

$$
\mathcal{H}_{\text {rel }} \Psi_{\text {rel }}=E_{\text {rel }} \Psi_{\text {rel }},
$$

where $\mathcal{H}_{\text {rel }}$ is given in (9). Taking into account the wave function in the form

$$
\Psi_{\text {rel }}(r, \varphi)=\frac{\mathrm{e}^{\mathrm{i} m_{z} \varphi}}{\sqrt{2 \pi}} r^{m_{z}} \mathrm{e}^{-\Omega r^{2} / 4} g(r),
$$

we obtain

$$
\begin{aligned}
& r^{2} g^{\prime \prime}+\left(2 m_{z}+1\right) r g^{\prime}-\frac{1}{\epsilon_{r}} r g \\
& \quad+\left(E_{b}-\Omega m_{z}-\Omega\right) r^{2} g-\Omega r^{3} g^{\prime}=0,
\end{aligned}
$$

where $E_{b}=E_{\text {rel }}-\frac{1}{2} \gamma m_{z}$. Equation (B14) has asymptotic solution

$$
g(r)=\text { const } \times r^{\eta}, \quad r \rightarrow \infty,
$$

where

$$
\eta=\frac{E_{b}}{\Omega}-m_{z}-1
$$

is, in general, a real number. This asymptotic behavior ensures square-integrability of the wave function (B13) and determines the asymptotic boundary condition

$$
\lim _{r \rightarrow \infty} \frac{g^{\prime}(r)}{g(r)}=\frac{\eta}{r}
$$

[2pt] For any energy, we look for solutions in the form of power series

$$
g(r)=\sum_{i=0}^{\infty} a_{i} r^{i}
$$

which leads to the recurrence relation for the coefficients,

$$
\begin{aligned}
& i\left(i+2 m_{z}\right) a_{i}-\epsilon_{r}^{-1} a_{i-1} \\
& \quad+\left[E_{b}-\Omega\left(m_{z}+i-1\right)\right] a_{i-2}=0,
\end{aligned}
$$

where $i=0,1,2, \ldots$ and $a_{j} \equiv 0$ for $j<0$. Solving the above relation we obtain the set of coefficients for any $E_{b}$. By imposing asymptotic boundary conditions (B17) at finite distance $r=\xi$ we can determine approximate energy eigenvalues from the nonlinear equation

$$
\Delta\left(E_{b}\right) \equiv \sum_{i=0}^{I}(i-\eta) a_{i}\left(E_{b}\right) \xi^{i}=0,
$$

where $I$ is a cut-off parameter. We note that the relative motion energies are given as $E_{\text {rel }}=E_{b}+\frac{1}{2} \gamma m_{z}$. The accuracy of energy increases with increasing $\xi$ and $I$. However, to achieve desired accuracy, highprecision arithmetic is required. The approximate radial amplitudes have the form

$$
G_{n m_{z}}(r)=\tilde{\mathcal{N}}_{n m_{z}} r^{m_{z}} \mathrm{e}^{-\Omega r^{2} / 4} \sum_{i=0}^{I} a_{i} r^{i},
$$

where $\tilde{\mathcal{N}}_{n m_{z}}$ is the normalization factor and $n$ is the eigenvalue number. One can note that when $\eta$ becomes positive integer $\left(\eta=n^{\prime}\right)$, then closed-form solutions exist. This means that the recurrence relation (B19) reduces to a finite system of $n^{\prime}+1$ linear equations. From the condition of linear dependence of the uniform system we determine values of $\Omega_{n^{\prime}}$ corresponding to exact solutions, for a given $n^{\prime}$. According to (B16) energies corresponding to exact solutions are $E_{b}^{n^{\prime}}=\Omega_{n^{\prime}}\left(m_{z}+n^{\prime}+1\right)$. Finally, the approximate expansion (B21) reduces exactly to a polynomial $\left(I=n^{\prime}\right)$. For example, if $n^{\prime}=1$, then closed-form solutions exist for confining frequencies 


$$
\Omega_{1}=\frac{1}{1+2 m_{z}} \epsilon_{r}^{-2}
$$

In this case the closed-form solutions read

$$
G_{m_{z}}^{1}(r)=\tilde{\mathcal{N}}_{m_{z}} r^{m_{z}}\left(1+\epsilon_{r} \Omega_{1} r\right) \mathrm{e}^{-\Omega_{1} r^{2} / 4},
$$

where $\tilde{\mathcal{N}}_{m_{z}}$ is the normalization factor.

The above exact solutions correspond to energies

$$
E_{b}^{1}=\frac{2+m_{z}}{1+2 m_{z}} \epsilon_{r}^{-2}
$$

We note that the power series expansion method has been applied in several contexts in order to solve both the Schrödinger and the Dirac equations with power potentials [34, 44-46].

\section{APPENDIX C: Radial integrals}

Matrix elements of the SO Hamiltonian read

$$
\begin{aligned}
& \square_{\mathrm{c} . \mathrm{m}}=\alpha \sqrt{2} \int_{0}^{\infty} F_{N^{\prime}, M_{z}+\Delta m_{z}}\left[\frac{\partial F_{N^{\prime}, M_{z}+\Delta m_{z}-1}}{\partial R}-\left(\frac{1}{2} \gamma R+\frac{M_{z}+\Delta m_{z}-1}{R}\right) F_{N^{\prime}, M_{z}+\Delta m_{z}-1}\right] R \mathrm{~d} R, \\
& \nabla_{\mathrm{c} . \mathrm{m}}=\alpha \sqrt{2} \int_{0}^{\infty} F_{N^{\prime}, M_{z}+\Delta m_{z}+1}\left[\frac{\partial F_{N^{\prime}, M_{z}+\Delta m_{z}}}{\partial R}-\left(\frac{1}{2} \gamma R+\frac{M_{z}+\Delta m_{z}}{R}\right) F_{N^{\prime}, M_{z}+\Delta m_{z}}\right] R \mathrm{~d} R, \\
& \mathbf{\square}_{\mathrm{rel}}=-\frac{\alpha \sqrt{2}}{2} \delta_{\Delta m_{z}, 1} \int_{0}^{\infty} F_{N, M_{z}} F_{N^{\prime}, M_{z}+\Delta m_{z}-1} R \mathrm{~d} R \int_{0}^{\infty} G_{n m_{z}}\left[\frac{\partial G_{n^{\prime} m_{z}^{\prime}}}{\partial r}-\left(\frac{1}{2} \gamma r+\frac{m_{z}^{\prime}}{r}\right) G_{n^{\prime} m_{z}^{\prime}}\right] r \mathrm{~d} r \\
& \mathbf{\Delta}_{\mathrm{rel}}=-\frac{\alpha \sqrt{2}}{2} \delta_{\Delta m_{z},-1} \int_{0}^{\infty} F_{N, M_{z}} F_{N^{\prime}, M_{z}+\Delta m_{z}+1} R \mathrm{~d} R \int_{0}^{\infty} G_{n m_{z}}\left[\frac{\partial G_{n^{\prime} m_{z}^{\prime}}}{\partial r}+\left(\frac{1}{2} \gamma r+\frac{m_{z}^{\prime}}{r}\right) G_{n^{\prime} m_{z}^{\prime}}\right] r \mathrm{~d} r,
\end{aligned}
$$

where $m_{z}=0, \pm 2, \pm 4, \ldots, m_{z}^{\prime}= \pm 1, \pm 3, \ldots$, $\Delta m_{z}=m_{z}-m_{z}^{\prime}$. The integrals marked by "c.m." are calculated analytically. The integrals marked by "rel" are calculated analytically for the case of noninteracting electrons and numerically for the case of interacting electrons.

\section{References}

[1] S.A. Wolf, D.D. Awschalom, R.A. Buhrman, J.M. Daughton, S. von Molnár, M.L. Roukes, A.Y. Chtchelkanova, D.M. Treger, Science 294, 1488 (2001).

[2] E.I. Rashba, Fiz. Tverd. Tela (Leningrad) 2, 1224 (1960).

[3] Y.A. Bychkov, E.I. Rasbha, Pisma Zh. Eksp. Teor. Fiz. 39, 66 (1984).

[4] R. Winkler, Spin-Orbit Coupling Effects in Two-Dimensional Electron and Hole Systems, Springer Tracts in Modern Physics, New York 2003.

[5] G. Bihlmayer, O. Rader, R. Winkler, New J. Phys. 17, 050202 (2015).

[6] J.H. Reina, L. Quiroga, N.F. Johnson, Phys. Rev. A 65, 032326 (2002).

[7] B. de Lima Bernardo, Phys. Lett. A 381 , 2239 (2017).

[8] A. Poszwa, Phys. Scr. 93, 025102 (2018).

[9] A. Poszwa, Physica E 99, 145 (2018).
[10] D. Vasilyev, F.O. Schumann, F. Giebels, H. Gollisch, J. Kirschner, R. Feder, Phys. Rev. B 95, 115134 (2017).

[11] O. Voskoboynikov, O. Bauga, C.P. Lee, O. Tretyak, J. Appl. Phys. 94, 5891 (2003).

[12] E. Tsitsishvili, G.S. Lozano, A.O. Gogolin, Phys. Rev. B 70, 115316 (2004).

[13] F.E. Meijer, A.F. Morpurgo, T.M. Klapwijk, T. Koga, J. Nitta, Phys. Rev. B 70, 201307 (2004).

[14] F.E. Meijer, A.F. Morpurgo, T.M. Klapwijk, J. Nitta, Phys. Rev. Lett. 94, 186805 (2005).

[15] S. Weiss, R. Egger, Phys. Rev. B 72 , 245301 (2005).

[16] S. Weiss, M. Thorwart, R. Egger, Europhys. Lett. 76, 905 (2006).

[17] M. Trif, V.N. Golovach, D. Loss, Phys. Rev. B 75, 085307 (2007).

[18] M. Glazov, E. Sherman, V. Dugaev, Physica E 42, 2157 (2010).

[19] S. Banerjee, J. Rowland, O. Erten, M. Randeria, Phys. Rev. X 4, 031045 (2014).

[20] S. Mironov, A. Buzdin, Phys. Rev. Lett. 118, 077001 (2017).

[21] L.G.G.V.D. da Silva, N. Studart, Phys. Rev. B 71, 113302 (2005).

[22] V.P. Amin, M.D. Stiles, Phys. Rev. B 94, 104420 (2016). 
[23] V.P. Amin, M.D. Stiles, Phys. Rev. B 94, 104419 (2016).

[24] X.-T. An, J.-J. Liu, Phys. Lett. A 372, 6790 (2008).

[25] Y. Yan, F. Jiang, H. Zhao, Phys. Lett. A 380, 277 (2016).

[26] J. Birman, R. Nazmitdinov, V. Yukalov, Phys. Rep. 526, 1 (2013).

[27] M. Paillard, X. Marie, P. Renucci, T. Amand, A. Jbeli, J.M. Gérard, Phys. Rev. Lett. 86, 1634 (2001).

[28] M. Wagner, U. Merkt, A.V. Chaplik, Phys. Rev. B 45, 1951 (1992).

[29] R.C. Ashoori, H.L. Stormer, J.S. Weiner, L.N. Pfeiffer, K.W. Baldwin, K.W. West, Phys. Rev. Lett. 71, 613 (1993).

[30] M. Bahar, F. Ungan, A. Soylu, Physica E 114, 113567 (2019).

[31] E. Tölö, A. Harju, Phys. Rev. B 79, 075301 (2009).

[32] Y. Nishi, Y. Tokura, J. Gupta, G. Austing, S. Tarucha, Phys. Rev. B 75, 121301 (2007).

[33] R.G. Nazmitdinov, N.S. Simonović, Phys. Rev. B 76, 193306 (2007).

[34] A. Poszwa, Few-Body Systems 57, 1127 (2016).

[35] R.G. Nazmitdinov, N.S. Simonović, J.M. Rost, Phys. Rev. B 65, 155307 (2002).

[36] I. Magnúsdóttir, V. Gudmundsson, Phys. Rev. B 61, 10229 (2000).

[37] J. Wang, D.Y. Xing, J. Phys. Condens. Matter 18, 10437 (2006).
[38] N.M. Gergs, S.A. Bender, R.A. Duine, D. Schuricht, Phys. Rev. Lett. 120, 017701 (2018).

[39] P. Kościk, H. Hassanabadi, Few-Body Systems 52, 189 (2012).

[40] J.-J.S. De Groote, J.E.M. Hornos, A.V. Chaplik, Phys. Rev. B 46, 12773 (1992).

[41] R. Hanson, L.P. Kouwenhoven, J.R. Petta, S. Tarucha, L.M.K. Vandersypen, Rev. Mod. Phys. 79, 1217 (2007).

[42] A.V. Khaetskii, Y.V. Nazarov, Phys. Rev. B 64, 125316 (2001).

[43] V.N. Golovach, A. Khaetskii, D. Loss, Phys. Rev. Lett. 93, 016601 (2004).

[44] A. Poszwa, A. Rutkowski, Phys. Rev. A 75, 033402 (2007).

[45] A. Poszwa, Phys. Rev. A 82, 052110 (2010).

[46] A. Poszwa, Phys. Scr. 84, 055002 (2011).

[47] H. Zhang, C.-X. Liu, X.-L. Qi, X. Dai, Z. Fang, S.-C. Zhang, Nat. Phys. 5, 438 (2009).

[48] C.F. Destefani, S.E. Ulloa, G.E. Marques, Phys. Rev. B 69, 125302 (2004).

[49] P.A. Maksym, T. Chakraborty, Phys. Rev. B 45, 1947 (1992).

[50] S. Takahashi, R.S. Deacon, K. Yoshida, A. Oiwa, K. Shibata, K. Hirakawa, Y. Tokura, S. Tarucha, Phys. Rev. Lett. 104, 246801 (2010).

[51] A.A. Kovalev, G.E.W. Bauer, A. Brataas, Phys. Rev. Lett. 94, 167201 (2005). 